Современные возможности ультразвуковых методов оценки атеросклеротического процесса у больных семейной гиперхолестеринемией

\author{
А.И. Ершова*, А.Н. Мешков, С.А. Бойцов, Т.В. Балахонова \\ ФГУ “Российский кардиологический научно-производственный комплекс” Министерства \\ здравоохранения и социального развития Российской Федерации. Москва, Россия
}

\title{
Modern ultrasound methods and atherosclerosis assessment in patients with familial hypercholesterolemia
}

\author{
A.I. Ershova*, A.N. Meshkov, S.A. Boytsov, T.V. Balakhonova \\ Russian Cardiology Scientific and Clinical Complex. Moscow, Russia
}

\begin{abstract}
Семейная гиперхолестеринемия (СГХС) - заболевание, сопровождающееся ранним развитием и быстрым прогрессированием атеросклероза. Учитывая, что распространенность СГХС в России растет, изучение методов диагностики атеросклероза у больных СГХС особенно актуально. Ультразвуковой метод исследования артерий - информативный, доступный, безопасный для оценки атеросклеротических изменений. В настоящее время имеющиеся методы ультразвукового исследования сосудов позволяют всесторонне описать стенку сосуда: дать структурную (качественную и количественную) и функциональную характеристики. Большая часть ультразвуковых параметров состояния артериальной стенки еще не нашла широкого применения в клинической практике, либо используется преимущественно в научных целях, либо находится на стадии разработки. Наиболее перспективными для применения в клинической практике у больных СГХС представляются маркеры, характеризующие одновременно степень выраженности и распространенности атеросклеротического процесса, такие как суммарная высота бляшек, лодыжечно-плечевой индекс, количество бляшек.
\end{abstract}

Ключевые слова: семейная гиперхолестеринемия, атеросклероз, атеросклеротическая бляшка, толщина комплекса интима-медиа, ультразвуковые исследования.

Familial hypercholesterolemia $(\mathrm{FHCH})$ is characterised by early atherosclerosis development and its fast progression. The prevalence of $\mathrm{FHCH}$ in Russia has been increasing, therefore, it is important to evaluate the potential of various methods for atherosclerosis diagnostics. Arterial ultrasound is an informative, accessible, and safe method for evaluating atherosclerotic vascular changes. The modern ultrasound methods allow describing vascular wall status in detail, including qualitative and quantitative characteristics of its structure and function. At the same time, most ultrasound characteristics of arterial wall are used in research settings or are under development, and not implemented into clinical practice. The most promising parameters, applicable for clinical use in FHCH patients, simultaneously describe atherosclerosis severity and its localization, such as total plaque height, ankle-brachial index, and plaque number.

Key words: Familial hypercholesterolemia, atherosclerosis, intima-media thickness, ultrasound methods.

с Коллектив авторов, 2011

e-mail: alersh@mail.ru

Тел.: 8(495) 414-65-96, 8 (916) 559-85-36.

[Ершова А.И. (*контактное лицо) - аспирант, лаборант-исследователь лаборатории ультразвуковых методов исследования сосудов, Мешков А.Н. - научный сотрудник отдела возрастных проблем сердечно-сосудистых заболеваний, Бойцов С.А. - руководитель отдела возрастных проблем сердечно-сосудистых заболеваний, Балахонова Т.В. - ведущий научный сотрудник отдела новых методов диагностики, руководитель лаборатории ультразвуковых методов исследования сосудов]. 
Семейная гиперхолестеринемия (СГХС) - жизнеугрожающее заболевание, характеризуюшееся повышенным уровнем холестерина липопротеинов низкой плотности (ХС ЛНП), что приводит к раннему появлению атеросклероза и повышенному риску раннего развития ишемической болезни сердца (ИБС) [47]. У нелеченных больных СГХС риск развития инфаркта миокарда (ИМ) к 50 годам составляет $51,4 \%$ для мужчин и $85,4 \%$ для женщин, к 60 годам - 12,2\% и 56,5\% соответственно [3]. СГХС отличается длительным бессимптомным течением, в связи с чем особенно важно диагностировать заболевание и оценивать степень (ст.) выраженности атеросклероза на ранних стадиях [47].

Ультразвуковой метод исследования сонных и бедренных артерий - неинвазивный, информативный, доступный, безопасный, относительно недорогой при сравнении с МСКТ, метод исследования сосудов, позволяющий у больных СГХС:

- проводить раннюю доклиническую диагностику атеросклероза,

- исследовать ст. выраженности атеросклеротического поражения сосудов в любом возрасте,

- наблюдать за атеросклеротическим процессом в динамике,

- оценивать эффективность терапии,

- оценивать риск коронарного атеросклероза $[10,31,42]$

В настоящее время имеются ультразвуковые маркеры атеросклероза, позволяющие дать как качественную и количественную структурную, так и функциональную характеристики стенки сосуда. В реальной клинической практике проводятся только дуплексное сканирование экстракраниального отдела брахицефальных артерий по стандартному протоколу, допплерография артерий нижних конечностей; при наличии значимых изменений кровотока возможно проведение дуплексного сканирования артерий нижних конечностей, реже измеряется лодыжечно-плечевой индекс (ЛПИ). Большая часть ультразвуковых параметров состояния артериальной стенки еще не нашла применения в клинической практике, либо используется преимущественно в научных целях, либо находится на стадии разработки.

\section{Распространенные в клинической практике ультразвуковые методы исследования сонных и бедренных артерий}

\section{Дуплексное сканирование артерий}

В широкой диагностической практике проводится стандартное дуплексное сканирование экстракраниального отдела брахицефальных и реже бедренных артерий, позволяющее оценивать атеросклеротические изменения качественно: наличие атеросклеротической бляшки (АБ), ее локализация, форма, подвижность, структура, плотность, состояние поверхности, наличие осложнений, и количественно: процент стеноза, скорость кровотока в области стеноза. АБ - фокальная структура, либо выступающая в просвет артерии не менее чем на $50 \%$ от величины толщины комплекса интима-медиа (ТКИМ) прилегающих участков артерии, либо имеющая толщину, измеренную как расстояние между линиями раздела “медиа-адвентиция" и “просвет артерии-интима", > 1,5 мм [56].

Одним из основных достоинств ультразвукового дуплексного сканирования сосудов является его способ- ность неинвазивно выявлять с высокой степенью точности малые $(<40 \%$ редукции диаметра сосуда), а также бессимптомные, еще не проявляющиеся клинически изменения. Дуплексное сканирование - неинвазивная прямая методика, позволяющая достаточно четко определить морфологию АБ и выявить осложнения (кровоизлияние, изъязвление), что чрезвычайно важно для определения тактики лечения больного и показаний к операции [1]. При исследовании 59 больных СГХС в возрасте $46,6 \pm 12,1$, у 44 из которых диагностировали каротидный атеросклероз, и было показано, что наличие гетерогенных АБ достоверно ассоциирует с ИБС [44]. Метод отличается зависимостью от оператора и позволяет описать только локальные атеросклеротические изменения.

Лодыжечно-плечевой индекс

ЛПИ - соотношение давления на лодыжке и давления на плече, определение которого впервые было выполнено с помощью ультразвукового исследования (УЗИ). Было показано, что ЛПИ $<0,9-$ независимый фактор, предсказывающий развитие серьезных сердечно-сосудистых осложнений (ССО) [38]. В 2006г появилось сообщение о результатах проспективного исследования на 1325 больных, длившегося 13 лет, позволяющих говорить о ЛПИ, как сильном прогностическом факторе развития ИМ и сердечно-сосудистой смерти. Было отмечено, что при снижении ЛПИ на 0,1 риск ИМ увеличивается на $22 \%$, а риск сердечно-сосудистой смерти на $35 \%$ [29]. ЛПИ - надежный, воспроизводимый, доступный клинической практике показатель, позволяющий даже на доклинической стадии выявить наличие гемодинамически значимой патологии артерий нижних конечностей, оценить состояние кровообращения, в т.ч. в динамике, с высокой долей вероятности предположить наличие атеросклеротических изменений в каротидном и коронарном бассейнах [2]. В исследовании [28] с помощью ЛПИ было выявлено 10-кратное превышение распространенности гемодинамически значимого атеросклероза артерий нижних конечностей среди больных СГХС (68 пациентов в возрасте 45,8 $\pm 11,6$ лет) по сравнению с контрольной группой (гр.) $(\mathrm{n}=27)$, сопоставимой по возрасту, полу, весу, курению и наличию артериальной гипертензии (АГ). В то же время ЛПИ нельзя считать универсальным методом оценки выраженности атеросклеротического процесса, т. к. этот показатель информативен только при наличии уже развившихся гемодинамически значимых изменений в сосудах нижних конечностей и отличается недостаточной специфичностью, например, при наличии сахарного диабета 2 типа (СД-2).

Согласно Российским рекомендациям ВНОК 2009 целесообразно использовать следующую схему УЗИ артериального русла у пациентов с нарушениями липидного обмена:

Всем лицам при первичном выявлении дислипидемии (ДЛП) показано проведение дуплексного сканирования экстракраниального отдела брахицефальных артерий.

При выявлении АБ в сонных или бедренных артериях у взрослых повторное исследование рекомендуется провести через полгода, при отсутствии изменения структуры и размеров АБ за этот период, динамическое наблюдение показано 1 раз в год.

У лиц с ДЛП и клиническими признаками недостаточности мозгового кровообращения необходимо дуплексное сканирование брахицефальных артерий для 


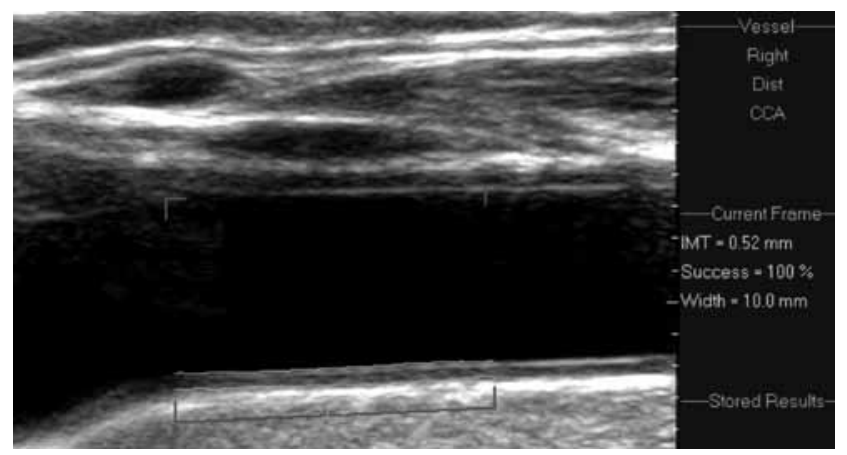

Puc. 1 Толщина КИМ у здорового человека (PHILIPS, iU-22, $\mathrm{L} 17,5 \mathrm{MHz})$.

исключения атеросклеротического поражения сосудов головного мозга: стеноз $>75 \%$, изъязвление, кровоизлияние в АБ, появление гипоэхогенных АБ.

Пациентам > 40 лет следует измерить ЛПИ.

В случае низких значений ЛПИ, а также при наличии $\geq 2$ факторов риска (ФР) даже при его исходно нормальных значениях измерение ЛПИ следует определять ежегодно.

Появление симптомов перемежающей хромоты требует исследования ЛПИ.

Детям > 10 лет с диагнозом СГХС показано измерение ТКИМ [3].

\section{Ультразвуковые методы, применяемые преимущественно в научных целях}

Толшина комплекса интима-медиа

ТКИМ в настоящее время является наиболее признанным показателем развития атеросклеротического процесса и ремоделирования сосудов, измеряемым и мониторируемым при проведении УЗИ сосудов. ТКИМ рассматривают как доказанный неинвазивный суррогатный маркер атеросклероза $[11,62]$.

Опубликованы рекомендации Американского общества эхокардиографии (АОЭ) 2008 по исследованию ТКИМ, в которых перечислены необходимые требования к аппаратуре, персоналу, методу проведения и интерпретации результатов исследования. Измерение средней ТКИМ проводится по задней стенке в дистальной трети общей сонной артерии (OCA) на расстоянии 1 см от бифуркации. В этих же рекомендациях представлены нормативные показатели в соответствии с полом и возрастом (рисунки 1, 2). Если значение ТКИМ $\geq 75$ перцентили, то сердечно-сосудистый риск (ССР) высокий. Если значение ТКИМ $>25$, но $<75$ перцентили, то ССР умеренный. Если ТКИМ $\leq 25$ перцентили, то ССР низкий [56].

В мета-анализе 8 исследований $(\mathrm{n}=37197)$ было показано, что увеличение ТКИМ на 0,1 мм ассоциировано с увеличением риска развития ИМ с $10 \%$ до $15 \%$, а риска развития инсульта (МИ) с $13 \%$ до $18 \%$ [33].

Согласно рекомендациям АОЭ 2008г измерение ТКИМ следует проводить не только пациентам с промежуточным ССР (согласно Фремингемской шкале), но и:

- всем лицам, у которых есть семейный анамнез раннего развития ССЗ у родственника первой степени родства: у мужчин в возрасте $<55$ лет, у женщин $<65$ лет;

- у лиц в возрасте < 60 лет с единственным, но отягчающим ФР, например, наследственная гиперли-

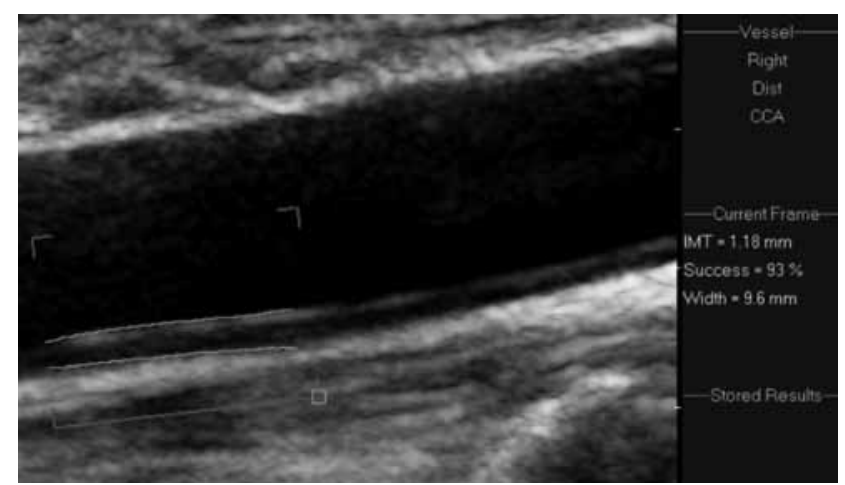

Puc. 2 Утолщение КИМ (PHILIPS, iU-22, L17,5MHz).

пидемия (ГЛП), у которых отсутствуют какие-либо другие показания к медикаментозной терапии атеросклероза;

• женщинам в возрасте $<60$ лет с $\geq 2$ ФР ССЗ [56].

Таким образом, больным с СГХС рекомендуется измерение ТКИМ.

Согласно ряду исследований утолщение ТКИМ как каротидных, так и бедренных артерий у больных СГХС ассоциируется с увеличением риска развития СС3 [65].

В мета-анализе 51 исследований проанализировали 4057 больных СГХС и показали, что по сравнению с лицами с нормальным уровнем ХС у больных СГХС средняя каротидная ТКИМ - стандартное отклонение 0,12 мм, $95 \%$ доверительный интервал (ДИ) 0,09-0,15 мм (р<0,001) и феморальная ТКИМ - 0,35 мм, 95 \% ДИ 0,180,51 мм $(\mathrm{p}<0,001)$ достоверно выше [34].

При сравнении 315 больных СГХС (11-67 лет) и 118 здоровых (11-76 лет), исследовали изменение ТКИМ у больных СГХС в зависимости от возраста. Было выявлено, что ежегодный прирост средней ТКИМ СА при СГХС достоверно выше (0,009 vs 0,004 мм/год), чем в гр. здоровых. В гр. здоровых ТКИМ достигает 0,78 мм к 76 годам, а в гр. больных СГХС к 40 годам [12]. Полученные результаты свидетельствуют о необходимости получения повозрастных норм для больных СГХС.

При исследовании 201 больного СГХС и 80 здоровых в возрасте 8-18 лет было показано, что средняя ТКИМ СА начинает увеличиваться уже в детском возрасте ТКИМ в гр. больных (0,494 мм) была значимо выше, чем у здоровых (0,472 мм) (p=0,002), причем достоверно значимые отличия ТКИМ в гр. больных СГХС наблюдались с возраста 12 лет. Также достоверно выше ежегодный прирост ТКИМ у детей, больных СГХС (0,005 vs 0,001 мм/ год) [64].

В одном из последних исследований была оценена взаимосвязь между типом мутации ЛНП-рецептора (ЛНП-р) и ТКИМ СА. Было исследовано 269 больных СГХС с нулевой аллелью гена ЛНП-р и 162 больныХ СГХС с дефектной аллелью гена ЛНП-р. При сопоставлении гр. по возрасту и полу различия между носителями нулевой и дефектной аллелей по уровню средней и максимальной ТКИМ КА выявлены не были. В то же время многофакторный анализ показал, что наличие нулевой аллели является независимым фактором повышения максимальной ТКИМ [24].

Ранее была найдена связь между типом генетического дефекта и выраженностью феморального атеросклероза, оцениваемого по величине средней ТКИМ бедренных артерий. Было показано, что средняя ТКИМ этих артерий увеличивается с возрастом в следующем порядке: нулевая 
аллель > дефектная аллель $>$ АРОВ $>$ контроль. Достоверно более высокая ТКИМ была отмечена в общей гр. носителей мутаций гена ЛНП-р по сравнению с АРОВ 3500 [25].

Измерение ТКИМ проводится не только для выявления атеросклеротического поражения, но и для оценки эффективности проводимого патогенетического лечения. УЗИ АСАРS (Asymptomatic Carotid Artery Plaque Study), KAPS (Kuopio Atherosclerosis Prevention Study), MARS (Monitored Atherosclerosis Regression Study), ARBITER (ARterial Bioligy for the Investigation of the Treatment Effects of Reducing), ASAP (Atorvastatin versus Simvastatin on Atherosclerosis Progression), REGRESS (Regression Growth Evaluation Statin Study), METEOR (Measuring Effects on intima media Thickness: an Evaluation Of Rosuvastatin) и др. убедительно показали, что эффективная гиполипидемическая терапия может остановить прирост ТКИМ, и даже привести к уменьшению этого параметра [16].

Среди пациентов с диагнозом СГХС также проводились крупные, проспективные, рандомизированные исследования эффективности гиполипидемической терапии, использующие ТКИМ в качестве конечной точки. К ним относятся исследование ASAP [49] и более поздние исследования RADIANCE I (Rating Atherosclerotic Disease change by Imaging with A New CETP inhibitor 1) [27], CAPTIVATE (Carotid Atherosclerosis Progression Trial Investigating Vascular ACAT inhibition Treatment Effects) [35], ENHANCE (Ezetimibe and Simvastatin in Hypercholesterolemia Enhances atherosclerosis regression) [26]. Следует заметить, что в исследования, в которых ТКИМ используется в качестве конечной точки, не рекомендуется включать пациентов с ранее проводимой интенсивной гиполипидемической терапией в связи с замедленным ростом ТКИМ на фоне длительного приема статинов [61].

Несмотря на широкое применение метода определения ТКИМ в научных целях (как в диагностических целях, так и для оценки эффективности проводимой терапии), ряд авторов указывают на недостатки и ограничения применения этого метода для оценки атеросклеротического процесса $[17,45]$. Во-первых, измерение ТКИМ, согласно последним рекомендациям, проводится в ОСА, что обусловлено большей доступностью места локации, перпендикулярным расположением артерии по отношению к лучу датчика и лучшей воспроизводимостью, в то время как образование АБ и прогрессирование ее роста обычно происходит в областях турбулентного потока, к которым относятся бифуркация и проксимальный сегмент внутренней СА (BCA). Таким образом, в процессы развития и прогрессирования атеросклероза в каротидном бассейне преимущественно вовлекаются бифуркация и ВСА, а не дистальный сегмент ОСА, в которой рекомендуется оценивать ТКИМ [50]. Во-вторых, сравнивать показатели ТКИМ предыдущих исследований сложно, т. к. методики измерения различались от исследования к исследованию: использовали только среднюю или только максимальную ТКИМ, измеряли ТКИМ задней стенки или усредняли значения ТКИМ задней и передней стенок ОСА или OCA, и бифуркации, и ВСА [11]. Вероятно, с появлением рекомендаций АОЭ 2008, описывающих методику измерения ТКИМ, и проведением исследований в соответствии с этими рекомендациями этот недостаток будет нивелирован [56]. Однако дискуссии по методу измерения ТКИМ продолжаются [11]. В-третьих, АБ, располагаю-

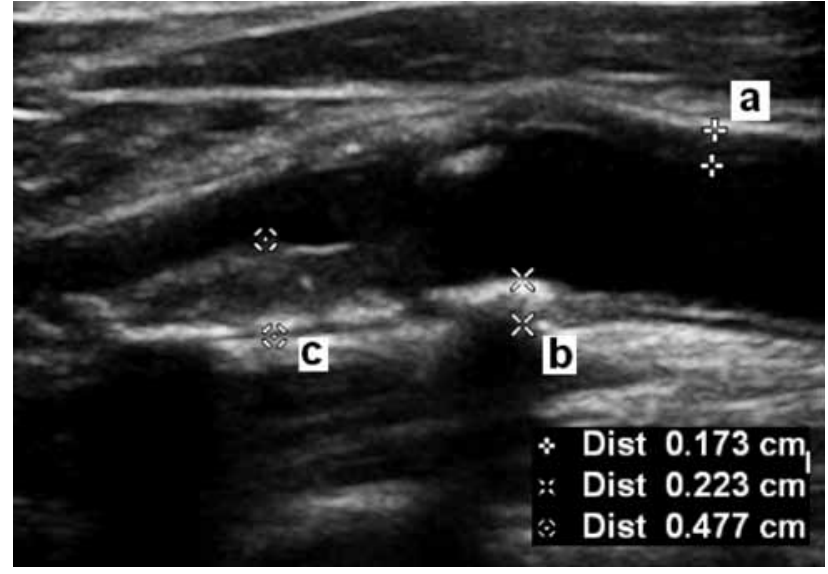

Puc. 3 Измерение показателя суммарной высоты АБ. PS=a+b+c. (PHILIPS, iU-22, L17,5MHz).

щаяся в том же сегменте артерии, где проводится оценка ТКИМ, не всегда может быть учтена. Измерение ТКИМ проводится только в трех сечениях (прямом, латеральном и заднем) и только задней (дальней от датчика) стенки, при этом толщина стенки в других участках этого сегмента артерии не оценивается, а именно в этих участках может быть утолщение КИМ и даже располагаться АБ [45]. В-четвертых, несмотря на то, что ТКИМ заявляют как суррогатный маркер, в действительности он соответствует не всем критериям понятия суррогатного маркера. Начатое в 2010г исследование PROG-IMT (Individual progression of carotid intima media thickness as a surrogate for vascular risk) (мета-анализ) призвано проверить соответствие ТКИМ этим критериям [32]. В-пятых, в ряде исследований показано, что ультразвуковые показатели, описывающие АБ: наличие АБ, процент стеноза, площадь АБ, объем АБ, в большей степени ассоциированы с ССР, чем ТКИМ [9,23]. Указывают, что существуют разные фенотипы атеросклероза, и ультразвуковые показатели, описывающие наличие и ст выраженности атеросклероза, отражают разные физиологические процессы, происходящие в стенке сосуда [53], а, возможно, и разные генетические варианты [39]. Например, увеличение ТКИМ может отражать гипертрофию медии, обусловленную наличием АГ. В то время как площадь поперечного сечения АБ, ее объем, кальциноз АБ могут дополнительно отражать процессы пролиферации пенистых клеток, фиброза и/или тромбоза [52]. При измерении средней ТКИМ, суммарной площади поперечного сечения АБ и суммарного объема АБ у 168 человек и анализ связи этих показателей с традиционными ФР, выявил, что ТКИМ достоверно ассоциировала с АГ, площадь АБ с курением и уровнем ОХС в плазме крови, а объем АБ с наличием СД [4]. Возможно, ТКИМ не является универсальным маркером атеросклероза и, вероятно, менее чувствительна для оценки атеросклеротического процесса, чем показатели, описывающие АБ.

Таким образом, в настоящее время ТКИМ наиболее изучена среди ультразвуковых маркеров атеросклероза, но чаще применяется в научных целях и пока не нашла широкого применения в клинической практике, т. к. требуют уточнения вопросы, касающиеся метода измерения ТКИМ, а также его чувствительности и специфичности.

\section{Суммарная высота АБ}

В последние годы все чаще обращаются к ультразвуковому показателю, называемому в англоязычной литературе как plaque score (PS). Сейчас термин еще не имеет 


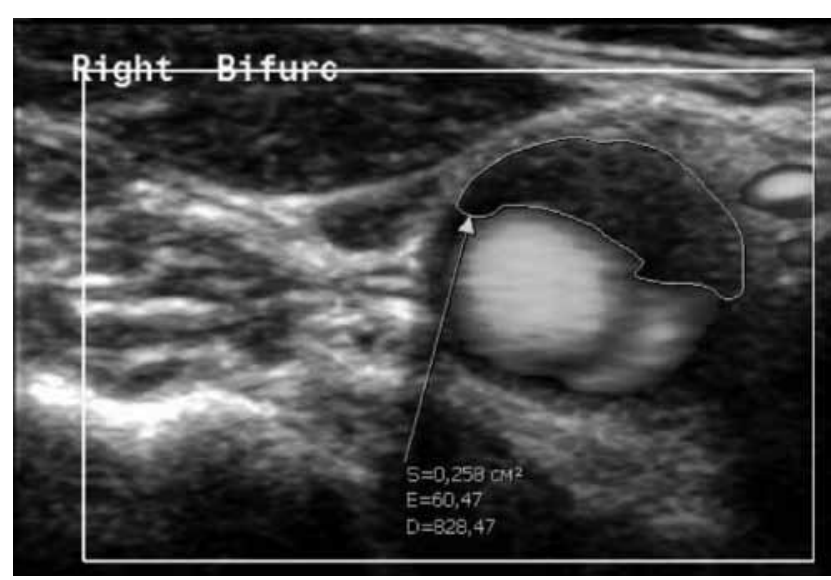

Puc. 4 Площадь (S) и гетерогенность (GSM=60,47) АБ с преобладанием компонентов средней плотности, измеренные в поперечном сечении (PHILIPS, iU-22, L17,5MHz; рабочая станция MultiVox2D, версия 1.17.0).

устоявшегося русского аналога. В этой работе он упоминается как суммарная высота как каротидных, так и феморальных АБ.

Также на сегодня не существует общепринятой методики определения PS. По данным разных авторов, PS сумма максимальной толщины всех АБ обеих СА (бедренных) $[20,37,58]$ либо отношение суммы максимальной толщины всех АБ обеих СА (бедренных) к количеству АБ [25]. Для расчета индекса артерию делят на 4 сегмента, каждый из которых протяженностью 1,5 см: 1 сегмент 1,5 см выше бифуркации, 2 сегмент - бифуркация, 3 и 4 сегменты - участки артерии ниже бифуркации. Максимальная толщина АБ измеряется на каждом из указанных сегментов [41] (рисунок 3).

У больных с уже развившимися АБ показатель суммарной толщины каротидных АБ, вероятно, более чувствительный маркер атеросклероза, чем ТКИМ ОСА. В исследовании [4] в гр. больных со стенозом KA $>50 \%(n=186)$ показатель суммарной толщины каротидных АБ был в 3 раза выше, чем в гр. без значимого стеноза KA $(n=84), \quad$ и составил $14,1 \pm 9,5$ и 4,6 $\pm 5,9$ соответственно. Более того, было получено, что PS и средняя ТКИМ бифуркации и ВСА сильнее ассоциированы с коронарными повреждениями, чем средняя ТКИМ ОСА, причем чувствительность и специфичность PS и средней ТКИМ бифуркации и ВСА были статистически сопоставимы, что свидетельствует о том, что PS такой же хороший предиктор коронарного атеросклероза, как и средняя ТКИМ бифуркации и ВСА, использующаяся в таких крупных исследованиях, как CHS (Cardiovascular Health Study) и ARIC (Atherosclerosis Risk In Communities) [41]. PS описывает состояние не только задней стенки дистальной трети ОСА, но и состояние передней стенки дистальной трети ОСА, и передних, и задних стенок еще 3 сегментов, в т.ч. бифуркации и ВСА, где АБ развиваются чаще и раньше по сравнению с OCA [17]. PS в отличие от средней ТКИМ ОСА отражает не локальные изменения, а в целом выраженность и распространенность атеросклеротического процесса в каротидном бассейне, т. е. атеросклеротическую нагруженность СА, но только при условии наличия АБ.

При сравнении PS у больных с коронарным атеросклерозом разной ст. выраженности и у больных без коро-

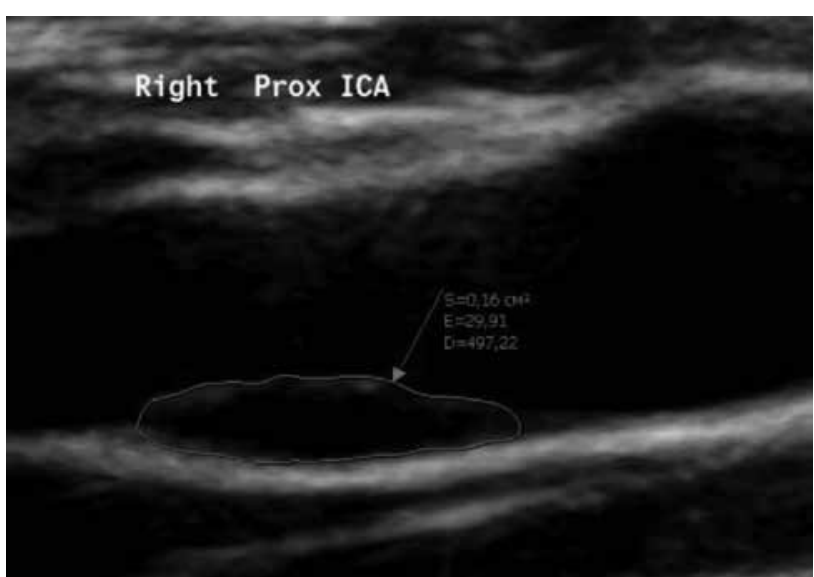

Puc. 5 Площадь (S) и гетерогенность $(\mathrm{GSM}=29,91)$ АБ низкой плотности, измеренные в продольном сечении (PHILIPS, iU-22, L17,5MHz; рабочая станция MultiVox2D, версия 1.17.0).

нарного атеросклероза (всего 116 пациентов) было показано, что суммарная высота каротидных АК > 1,9 является прогностическим значением, говорящим о наличии коронарного атеросклероза. При этом чувствительность метода составила 79,7 \%, а специфичность 63,4 \% [36].

При сравнении PS бедренных артерий у 123 больных с асимптомным течением СГХС и 23 больных СГХС с ранее перенесенным ИМ или нестабильной стенокардией (НС) было выявлено, что у больных СГХС с ранее перенесенным сердечно-сосудистым событием (CCC) PS в 5 раз достоверно выше, чем в гр. с асимптомным течением СГХС [25].

Показатель суммарной толщины каротидных АБ, вероятно, уже в ближайшем будущем будет рассматриваться как независимый предиктор наличия и тяжести ИБС [36]. Учитывая, что именно ИБС является основным клиническим проявлением СГХС, показатель суммарной высоты каротидных АБ, вероятно, может быть более чувствительным и специфичным для оценки ССР в этой гр. больных, но только при условии наличия АБ в СА.

В то же время, несмотря на положительные отзывы о методе определения суммарной толщины АБ, в исследованиях его используют редко, т. к. нет общепринятой методики расчета PS, недостаточно данных о воспроизводимости метода. Одна из проблем, с которой сталкиваются исследователи при расчете PS, это измерение ТКИМ передней стенки, которое оказывается неточным и маловоспроизводимым из-за особенностей формирования изображения при прохождении ультразвукового луча из более плотной структуры в менее плотную (слой адвентициа-медиа и слой интима-просвет) [56]. Для внедрения в клиническую практику и более широкого научного применения еще требуется дальнейшая оценки чувствительности и специфичности этого метода, что в свою очередь диктует необходимость проведения исследований на больших выборках в соответствии с концепцией доказательной медицины.

\section{Количество АБ}

В настоящее время отсутствует общепринятая методика расчета количества АБ. Если атеросклеротический процесс пролонгированный, и при этом АБ протяженные, переходят с одного сосуда на другой, то возникают вопросы, как определить границы между АБ и учесть их количество. В связи с этим нередко считают не коли- 
Таблица 1

Сравнительная характеристика методов УЗИ сосудов

\begin{tabular}{|c|c|c|c|c|}
\hline Параметры & Наличие доказательной базы & Информативность & Трудоемкость & Доступность \\
\hline Процент стеноза & ++ & ++ & + & +++ \\
\hline Линейная скорость кровотока & ++ & ++ & + & +++ \\
\hline лпи & +++ & ++ & ++ & +++ \\
\hline ТКИМ & +++ & ++ & + & +++ \\
\hline PS & ++ & ++ & ++ & ++ \\
\hline Количество АБ & ++ & ++ & + & +++ \\
\hline Площадь АБ & ++ & ++ & ++ & ++ \\
\hline Объем АБ & + & ++ & +++ & + \\
\hline ПЗВД & ++ & ++ & ++ & ++ \\
\hline Региональная артериальная жесткость & ++ & ++ & ++ & ++ \\
\hline Локальная артериальная жесткость & ++ & ++ & ++ & ++ \\
\hline Гетерогенность стенки сосуда (2D) & + & + & ++ & ++ \\
\hline Гетерогенность стенки сосуда (3D) & 0 & ++ & ++ & + \\
\hline Гетерогенность АБ & ++ & ++ & +++ & + \\
\hline
\end{tabular}

чество АБ непосредственно, а количество измененных сосудов.

В проспективном исследовании с участием 1325 больных продемонстрировано, что число артерий, пораженных АБ, варьировавшееся от 0 до 4 (поражение правой и левой СА, правой и левой бедренных артерий), является сильным прогностическим фактором развития ИМ и сердечно-сосудистой смерти [29]. В 4-летнем проспективном исследовании $(\mathrm{n}=367)$ отмечено увеличение относительного риска ОС и сердечно-сосудистой смертности с ростом числа каротидных АБ. При наличии 1-4 АБ риск ОС увеличивался в $\sim 3$ раза, при наличии $\geq 5$ АБ в $\sim 5$ раз [57].

В поисковой базе PubMed отсутствовали публикации, в которых бы сообщалось об определении количества АБ в СА (бедренных) у больных СГХС. Имеющиеся исследования, в которых оценивался этот показатель, свидетельствуют о его перспективности для изучения атеросклеротического процесса, в связи с чем представляется целесообразным использовать показатель количества АБ и при исследовании больных СГХС.

\section{Площадь АБ}

Единой методики измерения площади АБ на сегодня не существует. Площадь АБ оценивают либо в продольном сечении в плоскости наибольшего размера АБ либо в поперечном сечении в зоне максимального стеноза и рассчитывают суммарную площадь всех АБ [6] (рисунки $4,5)$.

Было показано повышение в 3,5 раза риска МИ и фатального ИМ в течение 5 лет у пациентов $(\mathrm{n}=1686)$ верхней квартили площади АБ по сравнению с пациентами нижней квартили, сопоставимыми по основным клинико-демографическим характеристикам [51].

В 6-летнем исследовании $(n=6226$ в возрасте 25-84 лет), в котором оценивалась чувствительность разных ультразвуковых показателей как предикторов первого ИМ, было показано, что у пациентов с площадью АБ СА 3 тертили (> 24,6 мм² для мужчин и $>18,7$ мм $^{2}$ для женщин) по сравнению с пациентами без каротидных АБ, сопоставимых по основным клинико-демографическим характеристикам, относительный риск первого ИМ достоверно возрастает как для мужчин, так и для женщин, но для женщин достоверно в большей степени, чем для мужчин $-1,56(1,04-2,36)$ и $3,95(2,16-7,19)$ соответственно. Причем показатель площади АБ был более сильным предиктором первого ИМ, чем ТКИМ - относительный риск для женщин при величине ТКИМ верхней квартили составил 2,86 $(1,07-7,65)$ [23].

Было показано, что площадь АБ увеличивается в 2,4 раза быстрее, нежели происходит увеличение ТКИМ. В связи с чем сделаны выводы, что показатель площади АБ более чувствительный маркер динамики атеросклеротического процесса, чем ТКИМ [6,55].

При оценке изменения площади продольного сечения АБ СА у 25 больных СГХС без гиполипидемической терапии за период 2-летнего наблюдения было получено достоверное увеличение площади АБ $-14,03 \pm 8,24$ vs

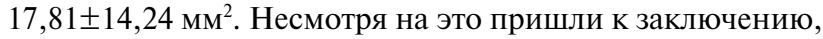
что возможности использования такого параметра атеросклеротических изменений, ограничены. Это связано с тем, что не у всех больных СГХС имеются АБ, площадь которых технически возможно измерить, что обусловлено их пролонгированностью, а также с отсутствием четких критериев измерения площади АБ, например, неясным остается вопрос границ АБ [63].

\section{Объем АБ}

Метод определения объема АБ представляет собой сканирование вдоль СА с получением поперечных срезов, которые далее реконструируются в 3D изображение.

Метод определения объема АБ перспективный и информативный, но не нашедший пока широкого распространения из-за своей трудоемкости и дороговизны. Учитывая тот факт, что рост АБ вдоль СА происходит в 2,4 раза быстрее, чем в сторону просвета артерии, метод определения объема АБ с помощью 3D изображения (как и площади АБ) представляется более чувствительным, чем измерение ТКИМ [54]. При исследовании 272 пациентов была оценена корреляционная связь между такими показателями, как ТКИМ, площадь и объем АБ. Наибольшая корреляционная связь была выявлена для показателей площади и объема АБ ( $\mathrm{R}$ составила 0,846), в связи с чем можно предположить, что метод определения площади АБ может заменить метод определения объема АБ. Как было представлено выше, эти показатели ассоциируют с разными традиционными ФР атеросклероза, что свидетельствует о том, что эти показатели, вероятно, представляют разные атеросклеротические 
фенотипы; каждый из этих показателей несет свою ценность для оценки атеросклеротического процесса [4]. Исследования, в которых бы оценивался объем АБ у больных СГХС, в настоящее время отсутствуют, но, вероятно, их появление - вопрос ближайшего будущего.

\section{Количественное исследование гетерогенности АБ в двухмерном режиме}

Для количественной оценки гетерогенности АБ разработан метод расчета медианы серой шкалы (GSM) в двухмерном изображении [19, 59] (рисунки 4, 5).

При количественном сравнении гетерогенности 81 симптомной и 111 асимптомных АБ показана, что симптомные АБ характеризуются гипоэхогенностью (медиана серой шкалы - 4), а асимптомные - гиперэхогенностью (медиана серой шкалы - 35) [59].

В многолетнем, популяционном исследовании сравнили метод визуальной оценки гетерогенности АБ и метод расчета медианы серой шкалы в 2-мерном режиме. Автоматический метод оценки гетерогенности АБ показал лучшую воспроизводимость по сравнению с визуальным методом, но сопровождался систематической ошибкой оценки, обусловленной проблемой стандартизации методики [18].

Выявление эндотелиальной дисфункщии сосудов

Эндотелиальная дисфункция (ЭД) - важный этап патогенеза атеросклероза [46]. В заявлении Рабочей группы по эндотелину и эндотелиальным факторам Европейского общества по гипертонии, 2005, подчеркивается, что для исследования функции эндотелия (ЭФ) необходимо использовать комплекс методов, включающий определение биохимических и ультразвуковых маркеров [13]. Неинвазивный тест для изучения ЭФ, основанный на реакции плечевой (или поверхностной бедренной) артерии на эндотелий-зависимый (поток-зависимый стимул в виде пробы с реактивной гиперемией) и эндотелий-независимый (сублингвальный прием нитроглицерина) стимулы с использованием ультразвука высокого разрешения, был предложен в 1992г [8]. В этом же исследовании на примере 10 детей, больных СГХС, в т.ч. 2 детей с гомозиготной формой СГХС, было показано, что эндотелий-зависимая вазодилатация (ЭЗВД) у больных СГХС снижена, в то время как эндотелий-независимая вазодилатация (ЭНВД) не была изменена [8]. Подобные результаты были получены и в работе [5]. Именно снижение ЭЗВД в отличие от ЭНВД в большинстве исследований ассоциировано с повышенным риском ИБС [60].

Поток-зависимая вазодилатация (ПЗВД) у больных с СГХС значительно снижена и колеблется по разным данным 2,2-4,9\%, тогда как у здоровых лиц этот параметр составляет 8-10 \% [46]. В мета-анализе результатов 51 исследований больных СГХС оценивалась не только ТКИМ, но и ПЗВД. Было показано, что у больных СГХС величина ПЗВД меньше, чем у лиц с нормальным уровнем ХС (стандартное отклонение составило -5,31\%, $95 \%$ ДИ $(-7,09)-(-3,53) \%(\mathrm{p}<0,001)$. Причем на фоне приема статинов наблюдалось улучшение (увеличение) ПЗВД $5,39 \%, 95$ \%ДИ 2,86 - 7,92 \% (p<0,001), что коррелировало с продолжительностью приема и дозой статинов [34].

Метод оценки ПЗВД неинвазивен, безопасен для пациента, воспроизводим. В то же время метод имеет ряд ограничений: во-первых, он не дает ответа на вопрос о том, что именно, ФР или уже существующее заболевание, приводит к снижению ПЗВД; во-вторых, на значение ПЗВД влияет ряд факторов, таких, как терапия вазоактивными препаратами, фолликулярная фаза менструального цикла, предшествующие исследованию прием жирной пищи или значительная $\Phi \mathrm{H}$; в-третьих, даже у здоровых лиц без ФР ССЗ существует разброс нормальных значений ПЗВД. В настоящее время определение ПЗВД - инструмент научных исследований, позволяющий мониторировать состояние сосудистой стенки при модификации ФР и на фоне лечения [1]. Возможности этого метода для использования в клинической практике, в т.ч. как маркера субклинического атеросклероза, требуют проведения дальнейших исследований [46].

\section{Исследование ригидности артерий}

Атеросклеротические изменения в артериальной стенке включают в себя пролиферацию гладкомышечных клеток (ГМК), отложение липидов, накопление коллагена, эластина и протеогликанов. Изменение соотношения коллагена и эластина приводит к изменению эластических свойств стенки сосуда [66].

Согласно заключению экспертов ЕОК 2006 по вопросу артериальной ригидности, измерение артериальной жесткости нужно рассматривать как рекомендованный тест для оценки ССР, особенно у тех пациентов, у которых при обычном обследовании не выявлены поражения органов-мишеней (ПОМ). “Золотым стандартом” измерения артериальной жесткости, по мнению экспертов, является аортальная скорость пульсовой волны (СПВ), определяемая при каротидно-феморальном положении датчиков [30].

Исследование артериальной жесткости как региональной (аортальной), так и локальной (каротидной и феморальной) возможно с помощью ультразвукового метода. В 1989г было показано, что у пациентов, перенесших ИМ, ригидность аорты - при трехсосудистом поражении коронарного русла параметр ригидности составил $22,37 \pm 4,29$, и СА $-13,17 \pm 4,56$, выше нормы $-8,58 \pm 3,02$ и $9,17 \pm 2,22$ соответственно, причем ригидность растет с числом пораженных сосудов, что позволило сделать вывод, что ригидность магистральных артерий может быть использована для прогноза ст. выраженности коронарного атеросклероза [22].

При оценке влияние липид-снижающей терапии на жесткость артерий у больных СГХС, у 45 больных

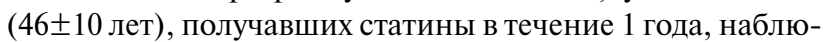
далось снижение жесткости стенки общих бедренных артерий, в то время как для ОСА подобные различия не обнаружены [48].

При сравнении показателей локальной ригидности СА у больных СГХС детского возраста $(n=18)$ и у здоровых детей $(n=18)$ было показано, что у детей с СГХС ригидность сосудистой стенки достоверно выше, чем у здоровых, причем для показателя ТКИМ различия получены не были, что свидетельствует, вероятно, о большей чувствительности параметров локальной ригидности по сравнению с ТКИМ для оценки атеросклеротических процессов у детей [40]. Предполагают, что количественная оценка ригидности артерий информативна до формирования видимых ультразвуковых структурных изменений.

Сегодня отсутствует достаточная доказательная база в отношении прогностического значения локальной ригидности ОСА, но исследования в этом направлении представляются перспективными. 
Методы, находящиеся на стадии разработки

Исследование гетерогенности КИМ

Липиды, накапливающиеся в стенке сосудов (в макрофагах и ГМК) в результате атеросклеротического процесса, могут быть визуализированы ультразвуковым методом в В-режиме и представляют собой локусы более высокой плотности [7].

В 2001г был предложен метод оценки гетерогенности стенки сосуда, КИМ, при котором рассчитывалась степень различий эхогенности в прилежащих друг к другу пикселях на участке 1 см от бифуркации ОСА. Предложенный показатель получил название гетерогенность стенки (WI - wall inhomogeneity). Авторы акцентировали внимание на том, что при оценке различий эхогенности в отличие от расчета абсолютных значений интенсивности отражения, получаемые результаты в меньшей степени зависят от настроек прибора [43].

В 2000г сначала был предложен, а затем в 2007г усовершенствован метод оценки гетерогенности КИМ. Авторы анализировали отношение интенсивности в гиперэхогенном слое и в точке, более отдаленной от просвета сосуда (в гипоэхогенном слое). Определяемый показатель получил название индекса гетерогенности КИМ (IMRI - intima-medial reflectivity index). Исследование проводилось у больных СГХС $(\mathrm{n}=30)$ в возрасте 35-77 лет. Определено, что при наличии утолщения КИМ > 0,8 мм и одновременно повышения IMRI >0,69 атеросклеротического СC3 выше, чем в случае наличия только повышенной ТКИМ. Авторы рассматривают IMRI как дополнительный информативный показатель раннего атеросклеротического процесса $[14,15]$.

В настоящее время ни один из предложенных методов оценки гетерогенности КИМ не получил дальнейшего развития, и не применяется даже в научных целях. Вероятно, это обусловлено, во-первых, низкой воспроизводимостью, что во многом связано с зависимостью ультразвукового изображения в серой шкале от настроек прибора, во-вторых, проведением измерений только в какой-либо одной плоскости, одной-двух проекциях, что не позволяет получать полную и объективную информацию о состоянии стенки сосуда.

Количественное исследование гетерогенности АБ в трехмерном режиме

Для количественной оценки гетерогенности АБ помимо метода расчета медианы серой шкалы в 2-мерном изображении разрабатывается метод расчета в 3-мерном режиме. Метод оценки гетерогенности АБ в 3-мерном режиме следует рассматривать как более информативный, чувствительный, но также и более дорогостоящий. Было показано, что АБ в СА, имеющие более низкую плотность, чаще наблюдаются у пациентов с симптоматическими АБ (стеноз $\geq 70 \%$ ), чем у пациентов с умеренной ст. стеноза $(<70 \%)$ [21]. Метод расчета медианы серой шкалы с помощью 3D УЗИ может быть полезным для оценки риска развития ишемии головного мозга у больных СГХС, но подобные исследования отсутствуют.

\section{Заключение}

В настоящее время методы УЗИ сосудов позволяют всесторонне описать стенку сосуда: дать структурную (качественную и количественную) и функциональную характеристику. При исследовании АБ возможно описать ее размеры (толщину, площадь, объем), структуру, ст. сужения ею просвета сосуда. Таким образом, имеются возможности комплексной оценки ст. атеросклеротических изменений. Но все эти методы отличаются по информативности, доступности, трудоемкости и доказательности. В таблице 1 дана сравнительная характеристика основных имеющихся методов УЗИ сосудов. Большая часть ультразвуковых маркеров атеросклероза применяется преимущественно в научных целях, но, вероятно, уже в ближайшем будущем с усовершенствованием технических возможностей и расширением доказательной базы для каждого из этих маркеров многие из них войдут в клиническую практиKy.

Самая большая клиническая база по применению ультразвуковых маркеров атеросклероза у больных СГХС, как и в целом в популяции, накоплена для ТКИМ и ЛПИ. Однако недостаточная информативность этих методов приводит к дальнейшему поиску ультразвуковых параметров как превосходящих указанные методы по информативности и воспроизводимости, так и дополняющих их. Появление универсального метода, описывающего всесторонне атеросклеротический процесс в сосудах вряд ли возможно, но представляется перспективной разработка комплексов параметров, разносторонне характеризующих системные и локальные особенности атеросклеротического процесса. У больных СГХС уже сегодня комплекс таких ультразвуковых параметров, как ПЗВД, ЛПИ, PS, количество АБ, позволяет судить об атеросклеротическом бремени артерий, что особенно актуально у больных СГХС, для которых характерно распространение атеросклеротического процесса не только в просвет сосуда, но и вдоль его стенки. Для локальной оценки атеросклероза перспективным представляется комплекс следующих методов: определение локальной жесткости артерий, объема АБ, гетерогенности структуры АБ, параметров нестабильности АБ.

Согласно исследованиям атеросклеротический процесс имеет разную морфологию в зависимости от преобладающего этиологического фактора развития атеросклероза с формированием различных его фенотипов. Следовательно, ультразвуковые маркеры атеросклероза могут отличаться в зависимости от фенотипа атеросклероза. Вероятно, СГХС также соответствует свой фенотип атеросклероза, и соответственно среди множества ультразвуковых маркеров есть тот, который будет более чувствительным и специфичным для больных СГХС.

Учитывая, что заболевание СГХС носит достаточно распространенный характер, в России выявлению и наблюдению больных СГХС уделяется все большее внимание, количество больных СГХС за счет улучшения диагностики будет расти. Ультразвуковые маркеры, специфичные для больных СГХС, оценивающие атеросклеротические изменения от субклинической стадии до тяжелой, а также характеризующие атеросклеротическое бремя артерий, позволят своевременно и качественно проводить диагностику, первичную и вторичную профилактику атеросклероза у больных СГХС. 


\section{Литература}

1. Балахонова Т.В. Ультразвуковая диагностика атеросклероза. Руководство по атеросклерозу и ишемической болезни сердца. Под ред. Акад. Е.И. Чазова, член-корр. РАМН В.В Кухарчика, проф. С.А. Бойцова. М.: Медиа Медика 2007; 736 c (159-75).

2. Рогоза А.Н., Балахонова Т.В., Чихладзе Н.М. и др Современные методы оценки состояния сосудов у больных артериальной гипертонией. М.: Издательский дом “Атмосфера" 2008; 72 с.

3. Диагностика и коррекция нарушений липидного обмена с целью профилактики и лечения атеросклероза Российские рекомендации IV пересмотра. ВНОК. Москва 2009.

4. Al-Shali K, House AA, Hanley AJ, et al. Differences between carotid wall morphological phenotypes measured by ultrasound in one, two and three dimensions. Atherosclerosis 2005; 178(2): 319-25.

5. Atkov OY, Balahonova TV, Pogorelova OA. Non-invasive ultrasound detection of endothelial dysfunction. Eur J Ultrasound 1998; 7(1): 37-45.

6. Barnett PA, Spence JD, Manuck SB, Jennings JR. Psychological stress and the progression of carotid artery disease. J Hypertens 1997; 15(1): 49-55.

7. Belcaro G, Barsotti A, Nicolaides AN. "Ultrasonic biopsy"--a non-invasive screening technique to evaluate the cardiovascular risk and to follow up the progression and the regression of arteriosclerosis. Vasa 1991; 20(1): 40-50.

8. Celermajer DS, Sorensen KE, Gooch VM, et al. Non-invasive detection of endothelial dysfunction in children and adults at risk of atherosclerosis. Lancet. 1992; 7; 340 (8828): 1111-5.

9. Chan SY, Mancini GB, Kuramoto L, et al. The prognostic importance of endothelial dysfunction and carotid atheroma burden in patients with coronary artery disease. JACC 2003 42(6): 1037-43.

10. Chimowitz MI, Weiss DG, Cohen SL, et al. Cardiac prognosis of patients with carotid stenosis and no history of coronary artery disease. Veterans Affairs Cooperative Study Group 167. Stroke 1994; 25(4): 759-65.

11. Cobble M, Bale B. Carotid intima-media thickness: knowledge and application to everyday practice. Postgrad Med 2010; 122(1): $10-8$.

12. de Groot E, Hovingh GK, Wiegman A, et al. Measurement of arterial wall thickness as a surrogate marker for atherosclerosis. Circulation 2004; 109(23 Suppl 1): III33-8.

13. Deanfield J, Donald A, Ferri C, et al. Endothelial function and dysfunction. Part I: Methodological issues for assessment in the different vascular beds: a statement by the Working Group on Endothelin and Endothelial Factors of the European Society of Hypertension. J Hypertens 2005; 23(1): 7-17

14. Ellis SM, Naoumova RP, Neuwirth CK, et al. Measurement of the reflectivity of the intima-medial layer of the common carotid artery improves the discriminatory value of intima-medial thickness measurement as a predictor of risk of atherosclerotic disease. Ultrasound Med Biol 2007; 33(7): 1029-38. Epub 2007 Apr 19.

15. Ellis SM, Sidhu PS. Granularity of the carotid artery intimamedial layer: reproducibility of quantification by a computerbased program. Br J Radiol 2000; 73(870): 595-600.

16. Espeland MA, O'leary DH, Terry JG, et al. Carotid intimalmedia thickness as a surrogate for cardiovascular disease events in trials of HMG-CoA reductase inhibitors. Curr Control Trials Cardiovasc Med 2005; 6(1): 3 .

17. Finn AV, Kolodgie FD, Virmani R. Correlation between carotid intimal/medial thickness and atherosclerosis: a point of view from pathology. Arterioscler Thromb Vasc Biol 2010; 30(2): 177 81. Epub 2009 Aug 13.
18. Fosse E, Johnsen SH, Stensland-Bugge E, et al. Repeated visual and computer-assisted carotid plaque characterization in a longitudinal population-based ultrasound study: the Troms study. Ultrasound Med Biol 2006; 32(1): 3-11.

19. Gronholdt ML, Nordestgaard BG, Wiebe BM, et al. Echolucency of computerized ultrasound images of carotid atherosclerotic plaques are associated with increased levels of triglyceride-rich lipoproteins as well as increased plaque lipid content. Circulation 1998; 97(1): 34-40.

20. Handa N, Matsumoto M, Maeda H, et al. Ultrasonic evaluation of early carotid atherosclerosis. Stroke 1990; 21(11): 1567-72.

21. Heliopoulos J, Vadikolias K, Mitsias P, et al. A three-dimensional ultrasonographic quantitative analysis of non-ulcerated carotid plaque morphology in symptomatic and asymptomatic carotid stenosis. Atherosclerosis 2008; 198(1):129-35. Epub 2008 Jan 24.

22. Hirai T, Sasayama S, Kawasaki T, Yagi S. Stiffness of systemic arteries in patients with myocardial infarction. A noninvasive method to predict severity of coronary atherosclerosis. Circulation 1989; 80(1): 78-86.

23. Johnsen SH, Mathiesen EB, Joakimsen O, et al. Carotid atherosclerosis is a stronger predictor of myocardial infarction in women than in men: a 6-year follow-up study of 6226 persons: the Troms Study. Stroke 2007; 38(11): 2873-80. Epub 2007 Sep 27.

24. Junyent M, Gilabert R, Jarauta E, et al. Impact of low-density lipoprotein receptor mutational class on carotid atherosclerosis in patients with familial hypercholesterolemia. Atherosclerosis 2010; 208(2): 437-41. Epub 2009 Aug 8.

25. Junyent M, Gilabert R, Zamb n D, et al. Femoral atherosclerosis in heterozygous familial hypercholesterolemia: influence of the genetic defect. Arterioscler Thromb Vasc Biol 2008; 28(3): 580-6. Epub 2007 Dec 20.

26. Kastelein JJ, Akdim F, Stroes ES, et al. Simvastatin with or without ezetimibe in familial hypercholesterolemia. $\mathbf{N}$ Engl $\mathbf{J}$ Med 2008; 358(14): 1431-43. Epub 2008 Mar 30.

27. Kastelein JJ, van Leuven SI, Burgess L, et al. Effect of torcetrapib on carotid atherosclerosis in familial hypercholesterolemia. N Engl J Med 2007; 356(16): 1620-30. Epub 2007 Mar 26.

28. Kroon AA, Ajubi N, van Asten WN, StalenhoefAF. The prevalence of peripheral vascular disease in familial hypercholesterolaemia. J Intern Med 1995; 238(5): 451-9.

29. Lamina C, Meisinger C, Heid IM, et al. Association of anklebrachial index and plaques in the carotid and femoral arteries with cardiovascular events and total mortality in a populationbased study with 13 years of follow-up. Eur Heart J 2006; 27(21): 2580-7.

30. Laurent S, Cockcroft J, Van Bortel L, et al. Expert consensus document on arterial stiffness: methodological issues and clinical applications. Eur Heart J 2006; 27(21): 2588-605. Epub 2006 Sep 25.

31. Leng GC, Papacosta O, Whincup P, et al. Femoral atherosclerosis in an older British population: prevalence and risk factors. Atherosclerosis 2000; 152(1): 167-74.

32. Lorenz MW, Bickel H, Bots ML, et al. Individual progression of carotid intima media thickness as a surrogate for vascular risk (PROG-IMT): Rationale and design of a meta-analysis project. Am Heart J 2010; 159(5): 730-6.e2.

33. Lorenz MW, Markus HS, Bots ML, et al. Prediction of clinical cardiovascular events with carotid intima-media thickness: a systematic review and meta-analysis. Circulation 2007; 115(4): 459-67.

34. Masoura C, Pitsavos C, Aznaouridis K, et al. Arterial endothelial function and wall thickness in familial hypercholesterolemia and familial combined hyperlipidemia and the effect of statins. A systematic review and meta-analysis. Atherosclerosis 2010. 
35. Meuwese MC, de Groot E, Duivenvoorden R, et al. ACAT inhibition and progression of carotid atherosclerosis in patients with familial hypercholesterolemia: the CAPTIVATE randomized trial. JAMA 2009; 301(11): 1131-9.

36. Morito $\mathrm{N}$, Inoue $\mathrm{Y}$, Urata $\mathrm{M}$, et al. Increased carotid artery plaque score is an independent predictor of the presence and severity of coronary artery disease. J Cardiol 2008; 51(1): 25-32.

37. Nagai Y, Kitagawa K, Sakaguchi M, et al. Significance of earlier carotid atherosclerosis for stroke subtypes. Stroke 2001; 32(8): 1780-5.

38. Papamichael CM, Lekakis JP, Stamatelopoulos KS, et al. Ankle-brachial index as a predictor of the extent of coronary atherosclerosis and cardiovascular events in patients with coronary artery disease. Am J Cardiol 2000; 86(6): 615-8.

39. Pollex RL, Hegele R. Genetic determinants of carotid ultrasound traits. Curr Atheroscler Rep 2006; 8(3): 206-15.

40. Riggio S, Mandraffino G, Sardo MA, et al. Pulse wave velocity and augmentation index, but not intima-media thickness, are early indicators of vascular damage in hypercholesterolemic children. Eur J Clin Invest 2010; 40(3): 250-7.

41. Sakaguchi M, Kitagawa K, Nagai Y, et al. Equivalence of plaque score and intima-media thickness of carotid ultrasonography for predicting severe coronary artery lesion. Ultrasound Med Biol 2003; 29(3): 367-71.

42. Salonen JT, Salonen R. Ultrasonographically assessed carotid morphology and the risk of coronary heart disease. Arterioscler Thromb 1991; 11(5): 1245-9.

43. Sandrock M, Cheng DC, Schmitz D, Schmidt-Trucks ss A Quantification of the wall inhomogeneity in B-mode sonographic images of the carotid artery. J Ultrasound Med 2002; 21(12): 1395-404.

44. Sidhu PS, Naoumova RP, Maher VM, et al. The extracranial carotid artery in familial hypercholesterolaemia: relationship of intimal-medial thickness and plaque morphology with plasma lipids and coronary heart disease. J Cardiovasc Risk 1996; 3(1): 61-7.

45. Simon A, Megnien JL, Chironi G. The value of carotid intimamedia thickness for predicting cardiovascular risk. Arterioscler Thromb Vasc Biol 2010; 30(2): 182-5. Epub 2009 Nov 30.

46. Simons LA, Sullivan D, Simons J, Celermajer DS. Effects of atorvastatin monotherapy and simvastatin plus cholestyramine on arterial endothelial function in patients with severe primary hypercholesterolaemia. Atherosclerosis 1998; 137(1): 197-203.

47. Slack J. Risks of ischaemic heart-disease in familial hyperlipoproteinaemic states. Lancet 1969; 2(7635): 1380-2.

48. Smilde TJ, van den Berkmortel FW, Wollersheim $\mathrm{H}$, et al. The effect of cholesterol lowering on carotid and femoral artery wall stiffness and thickness in patients with familial hypercholesterolaemia. Eur J Clin Invest 2000; 30(6): 473-80.

49. Smilde TJ, van Wissen S, Wollersheim H, et al. Effect of aggressive versus conventional lipid lowering on atherosclerosis progression in familial hypercholesterolaemia (ASAP): a prospective, randomised, double-blind trial. Lancet 2001; 357(9256): 577-81.

50. Solberg LA, Eggen DA. Localization and sequence of development of atherosclerotic lesions in the carotid and vertebral arteries. Circulation 1971; 43(5): 711-24.

51. Spence JD, Eliasziw M, DiCicco M, et al. Carotid plaque area: a tool for targeting and evaluating vascular preventive therapy.
Stroke 2002; 33(12): 2916-22.

52. Spence JD, Hegele RA. Non-invasive assessment of atherosclerosis risk. Curr Drug Targets Cardiovasc Haematol Disord 2004; 4(2): 125-8.

53. Spence JD, Hegele RA. Noninvasive phenotypes of atherosclerosis: similar windows but different views. Stroke 2004; 35(3): 649-53. Epub 2004 Feb 12.

54. Spence JD. Measurement of intima-media thickness vs. carotid plaque: uses in patient care, genetic research and evaluation of new therapies. Int J Stroke 2006; 1(4): 216-21.

55. Spence JD. Ultrasound measurement of carotid plaque as a surrogate outcome for coronary artery disease. Am J Cardiol 2002; 89(4A): 10B-15B; discussion 15B-6.

56. Stein JH, Korcarz CE, Hurst RT, et al. Use of carotid ultrasound to identify subclinical vascular disease and evaluate cardiovascular disease risk: a consensus statement from the American Society of Echocardiography Carotid Intima-Media Thickness Task Force. Endorsed by the Society for Vascular Medicine. J Am Soc Echocardiogr 2008; 21(2): 93-111.

57. St rk S, van den Beld AW, von Schacky C, et al. Carotid artery plaque burden, stiffness, and mortality risk in elderly men: a prospective, population-based cohort study. Circulation 2004; 110(3): 344-8. Epub 2004 Jul 6.

58. Takiuchi S, Kamide K, Miwa Y, et al. Diagnostic value of carotid intima-media thickness and plaque score for predicting target organ damage in patients with essential hypertension. J Hum Hypertens 2004; 18(1): 17-23.

59. Tegos TJ, Kalomiris KJ, Sabetai MM, et al. Significance of sonographic tissue and surface characteristics of carotid plaques. AJNR Am J Neuroradiol 2001; 22(8): 1605-12.

60. Urbina EM, Williams RV, Alpert BS, et al. Noninvasive assessment of subclinical atherosclerosis in children and adolescents: recommendations for standard assessment for clinical research: a scientific statement from the American Heart Association. Hypertension 2009; 54(5): 919-50. Epub 2009 Sep 3.

61. Vergeer M., Zhou R., Bots M.L. et al. Carotid atherosclerosis progression in familial hypercholesterolemia patients: a pooled analysis of the ASAP, ENHANCE, RADIANCE 1, and CAPTIVATE studies. Circ Cardiovasc Imaging. $2010 \mathrm{Jul}$ 1;3(4):398-404. Epub 2010 May 21.

62. Verhamme P, Kerkhof F, Buysschaert I, et al. Carotid intimamedia thickness: more than a research tool? Acta Cardiol 2010; 65(1): 59-66

63. Wendelhag I, Wiklund O, Wikstrand J. On quantifying plaque size and intima-media thickness in carotid and femoral arteries. Comments on results from a prospective ultrasound study in patients with familial hypercholesterolemia. Arterioscler Thromb Vasc Biol 1996; 16(7): 843-50.

64. Wiegman A, de Groot E, Hutten BA, et al. Arterial intimamedia thickness in children heterozygous for familial hypercholesterolaemia. Lancet 2004; 363(9406): 369-70.

65. Wittekoek ME, de Groot E, Prins MH, et al. Differences in intima-media thickness in the carotid and femoral arteries in familial hypercholesterolemic heterozygotes with and without clinical manifestations of cardiovascular disease. Atherosclerosis 1999; 146(2): 271-9.

66. Zieman SJ, Melenovsky V, Kass DA. Mechanisms, pathophysiology, and therapy of arterial stiffness. Arterioscler Thromb Vasc Biol 2005; 25(5): 932-43. Epub 2005 Feb 24. 